\title{
The evolving role and utility of off-label drug use in multiple myeloma
}

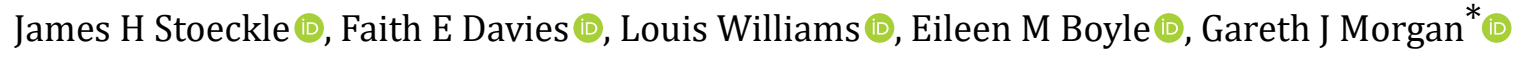 \\ Perlmutter Cancer Center, New York University Langone Health, New York, NY 10016, USA \\ *Correspondence: Gareth Morgan, Department of Medicine, NYU Grossman School of Medicine, Perlmutter Cancer Center, \\ New York University Langone Health, New York City, NY 10016, USA. gareth.morgan@nyulangone.org \\ Academic Editor: Luca Agnelli, IRCCS National Cancer Institute (NCI), Italy
}

Received: February 2, 2021 Accepted: March 16, 2021 Published: August 30, 2021

Cite this article: Stoeckle JH, Davies FE, Williams L, Boyle EM, Morgan GJ. The evolving role and utility of off-label drug use in multiple myeloma. Explor Target Antitumor Ther. 2021;2:355-73. https://doi.org/10.37349/etat.2021.00050

\begin{abstract}
The treatment landscape for multiple myeloma (MM) has dramatically changed over the last three decades, moving from no US Food and Drug Administration approvals and two active drug classes to over 19 drug approvals and at least eight different active classes. The advances seen in MM therapy have relied on both a structured approach to obtaining new labels and cautious off-label drug use. Although there are country and regional differences in drug approval processes, many of the basic principles behind off-label drug use in MM can be summarized into four main categories: 1) use of a therapy prior to the current approval regulations; 2) widespread use of a therapy following the release of promising clinical trial results but prior to drug approval; 3) use of a cheap therapy supported by clinical safety and efficacy data but without commercial backing; and 4) niche therapies for small well-defined patient populations where large clinical trials with sufficient power may be difficult to perform. This review takes a historical approach to discuss how off-label drug use has helped to shape the current treatment approach for MM.
\end{abstract}

\section{Keywords}

Multiple myeloma, off-label, drug development, orphan drugs, thalidomide, relapsed refractory

\section{Introduction}

There are currently 19 drugs approved by the US Food and Drug Administration (FDA) for the treatment of multiple myeloma (MM) [1] (Table 1). This portfolio represents a marked increase since the 1990s, when the only therapies available were alkylating agents—such as melphalan and cyclophosphamide-corticosteroids, and autologous stem cell transplantation (ASCT)-none of which had been formally reviewed by the FDA. Although the process for drug approval and widespread clinical use varies depending on geographical region, the basic principles behind requiring regulatory permission are now universal and important to ensure the safety and efficacy of new therapies.

However, many therapeutic advances have not only relied on this structured approach to obtaining new approvals but have also utilized off-label drug use. For example, in the US, once approved by the FDA healthcare providers may prescribe a drug for an indication outside of those included in the FDA label when 
they judge that it is medically appropriate. The history of drug development in MM over the last 30 years provides a striking example of how the common prescribing practice of off-label drug use [2-5] is used within a single disease setting, how this practice impacts the introduction of novel therapies, results in varied clinical trial designs, and eventually modifies clinical practice.

Table 1. List of drugs used in MM with FDA approval and labeled indications

\begin{tabular}{|c|c|c|c|}
\hline Novel agents & Year approved & $\begin{array}{l}\text { Indication } \\
\text { (lines of therapy failed) }\end{array}$ & Approved in combination* \\
\hline Thalidomide & 1998 & NDMM & Dexamethasone \\
\hline \multirow[t]{2}{*}{ Bortezomib (IV and SC) } & 2003 & NDMM & Melphalan/prednisone \\
\hline & & RRMM & \\
\hline \multirow[t]{2}{*}{ Lendalidomide } & 2005 & RRMM & Dexamethasone \\
\hline & & Maintenance & \\
\hline \multirow[t]{3}{*}{ Carfilzomib } & 2012 & $\operatorname{RRMM}(1-3)^{* *}$ & Lenalidomide/dexamethasone \\
\hline & & & Daratumumab/dexamethasone \\
\hline & & & Dexamethasone \\
\hline Pomalidomide & 2013 & RRMM (2) & Dexamethasone \\
\hline Panobinostat & 2015 & RRMM (2) & Bortezomib/dexamethasone \\
\hline \multirow[t]{7}{*}{ Daratumumab (IV and SC) } & 2015 & NDMM & Lenalidomide/dexamethasone \\
\hline & & & Bortezomib/melphalan/prednisone \\
\hline & & & Bortezomib/melphalan/dexamethasone \\
\hline & & RRMM (1) & Lenalidomide/dexamethasone \\
\hline & & & Bortezomib/dexamethasone \\
\hline & & $\operatorname{RRMM}(1-3)^{* *}$ & Carfilzomib/dexamethasone \\
\hline & & RRMM (2) & Pomalidomide/dexamethasone \\
\hline \multirow[t]{2}{*}{ Elotuzumab } & 2015 & RRMM & Lenalidomide/dexamethasone \\
\hline & & & Pomalidomide/dexamethasone \\
\hline Ixazomib & 2015 & RRMM & Lenalidomide/dexamethasone \\
\hline \multirow[t]{2}{*}{ Selinexor } & 2019 & RRMM (1) & Bortezomib/dexamethasone \\
\hline & & RRMM (PR) & Dexamethasone \\
\hline Belantamabmafodotin & 2020 & RRMM (4) & \\
\hline Isatuximab & 2020 & RRMM & Pomalidomide/dexamethasone \\
\hline \multicolumn{4}{|l|}{ Cytotoxic chemotherapy } \\
\hline Cyclophospha-mide (IV and PO) & 1959 & MM (unspecified) & \\
\hline \multirow[t]{2}{*}{ Melphalan (IV and PO) } & 1964 & Palliative & \\
\hline & & ASCT & \\
\hline Carmustine & 1977 & Palliative & Prednisone \\
\hline Liposomal doxorubicin & 1995 & RRMM (1) & Bortezomib \\
\hline \multicolumn{4}{|l|}{ Adjunctive therapy } \\
\hline Zoledronic acid & 1964 & & \\
\hline Pamidronate & 1995 & & \\
\hline Plerixafor & 2008 & & \\
\hline
\end{tabular}

* If no drugs listed in table under Approved in combination then approval was for use as single agent; ${ }^{*}$ FDA approval includes use as single agent. PR: penta-refractory; IV: intravenous; SC: subcutaneous; PO: oral; NDMM: newly diagnosed multiple myeloma; RRMM: relapsed/refractory multiple myeloma. Check FDA website and company prescribing for up-to-date details

The potential reasons for non-approved use can be summarized into four main categories: 1) use of a therapy prior to the current regulations; 2) widespread use of a therapy following the release of promising clinical trial results but prior to drug approval; 3) use of a cheap therapy supported by clinical safety and efficacy data but without commercial backing; and 4) niche therapies for small well-defined patient populations where large clinical trials with sufficient power may be difficult to perform (Figure 1). Regulatory approvals change frequently and as discussed above are country specific. This review will mainly discuss US 
FDA approvals, and although the specifics of a drug's label and its off-label use may differ by region, the basic principles behind off-label drug use are universal.

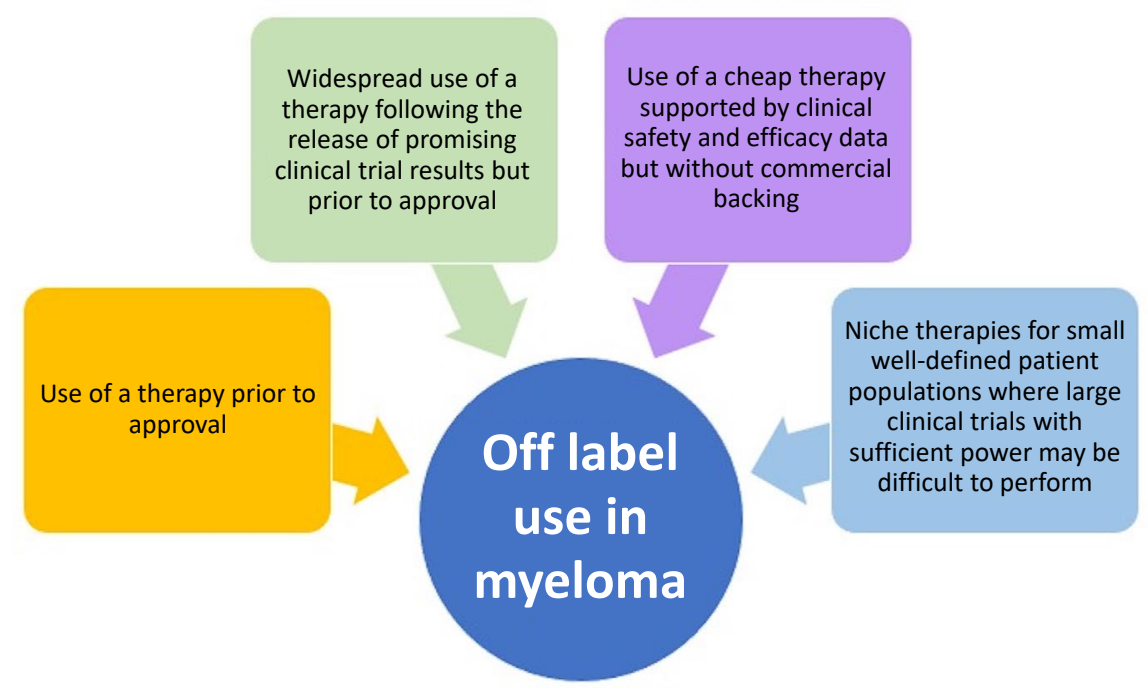

Figure 1. Reasons for off-label drug use in MM

\section{Drugs in regular use in MM without an FDA label}

Prior to the current MM therapeutic landscape, where multiple FDA-approved treatment options are available, the predominant drugs in use were corticosteroids and alkylating agents for which there were no formal approvals. Following the introduction of low doses of the alkylating agent melphalan, high-dose melphalan (HDM) was developed [6]. The toxicity of HDM was subsequently modified by the use of stem cell rescue with ASCT which improved time to blood count recovery and improved rates of infection and mortality [7, 8]. Although the escalating doses of melphalan were associated with high toxicity, for the first time, therapy in MM was associated with deep responses and healing of bone lesions.

The widespread uptake of HDM with ASCT was based on the results of a series of three studies which randomized standard dose chemotherapy against ASCT [9-11]. These trials showed improved progressionfree survival (PFS) compared with ASCT with a potential benefit for overall survival (OS). Importantly, HDM with ASCT changed the aim of therapy from the initial goal of disease control to one which recognized that deep responses translated into improved outcomes $[12,13]$. The doses of the alkylator cyclophosphamide and its associated drug combinations used most commonly in clinical practice are not FDA-approved. Off-label use of cyclophosphamide in MM is broad and includes MM renal impairment or amyloid, or as a cheaper third drug in a triplet combination $[14,15]$.

Corticosteroids have served as a critical backbone in the treatment of MM, from the early era to the current day. Dexamethasone is active as a single agent [16] and as part of drug combinations. The FDA label has a nonspecific indication for use in combination with other myeloma-directed therapies [17]. A series of studies showed dexamethasone improved response rates that were not maintained long term $[18,19]$, but it remained a backbone drug because of the importance of response rates during the drug approval process. Prednisone, which lacks an FDA label for MM, was the corticosteroid of choice when used in combination with low dose melphalan in the combination melphalan-prednisone (MP) [20], whereas dexamethasone was preferentially used with vincristine and doxorubicin as vincristine-doxorubicin-dexamethasone (VAD) [21]. Maximizing the dexamethasone dose was associated with better responses and a dose of $40 \mathrm{mg}$ daily on days 1-4, 9-12, and 17-20 became a standard in MM [22]. In this setting $70 \%$ of patients resistant to melphalan and $33 \%$ of those resistant to both melphalan and doxorubicin were noted to have a response. Later co-operative group studies of dexamethasone combinations with immunomodulatory drugs [23] (see below) highlighted some of the infections associated with dexamethasone. Refined dosing emerged, with once weekly dosing becoming more common (e.g., $40 \mathrm{mg}$ ) and a lower dose for older, less fit patients (e.g., 10-20 mg) to minimize infective and neurologic consequences. 
The cytotoxic chemotherapeutics vincristine and doxorubicin used in VAD still lack an FDA label. Though the liposomal form of doxorubicin has been approved, it is no longer in widespread use in the clinic [24]. These agents have been successfully combined with novel agents to generate the Velcade-thalidomidedexamethasone-cisplatin-doxorubicin-cyclophosphamide-etoposide (VTD-PACE) regimen (bortezomib, thalidomide, dexamethasone, cisplatin, doxorubicin, cyclophosphamide, etoposide). Like vincristine and doxorubicin, the cytotoxic agents etoposide, cisplatin, and cyclophosphamide used in the VTD-PACE regimen still do not have an FDA label for MM. Despite this they are widely used for patients with resistant and refractory disease where good responses are generated [25], and in the upfront setting as part of the total therapy regimens where excellent responses and clinical outcomes are seen [26].

\section{The novel drug era}

The development of the proteasome inhibitor (PI) bortezomib, and the immunomodulatory inhibitory drug (IMiD) lenalidomide in MM illustrates how new drugs were introduced into a setting where the primary therapies in use did not have an FDA label and how off-label drug use plays an important role in the drug development process. For both bortezomib and lenalidomide the initial challenge was to choose the most appropriate control drug for comparison with novel agents in the relapsed clinical setting where there was an unmet need for new therapies. Single agent dexamethasone, without an FDA label for this indication, was chosen over alkylating agents because of its ease of use and less myelosuppressive side effect profile (Figure 2).

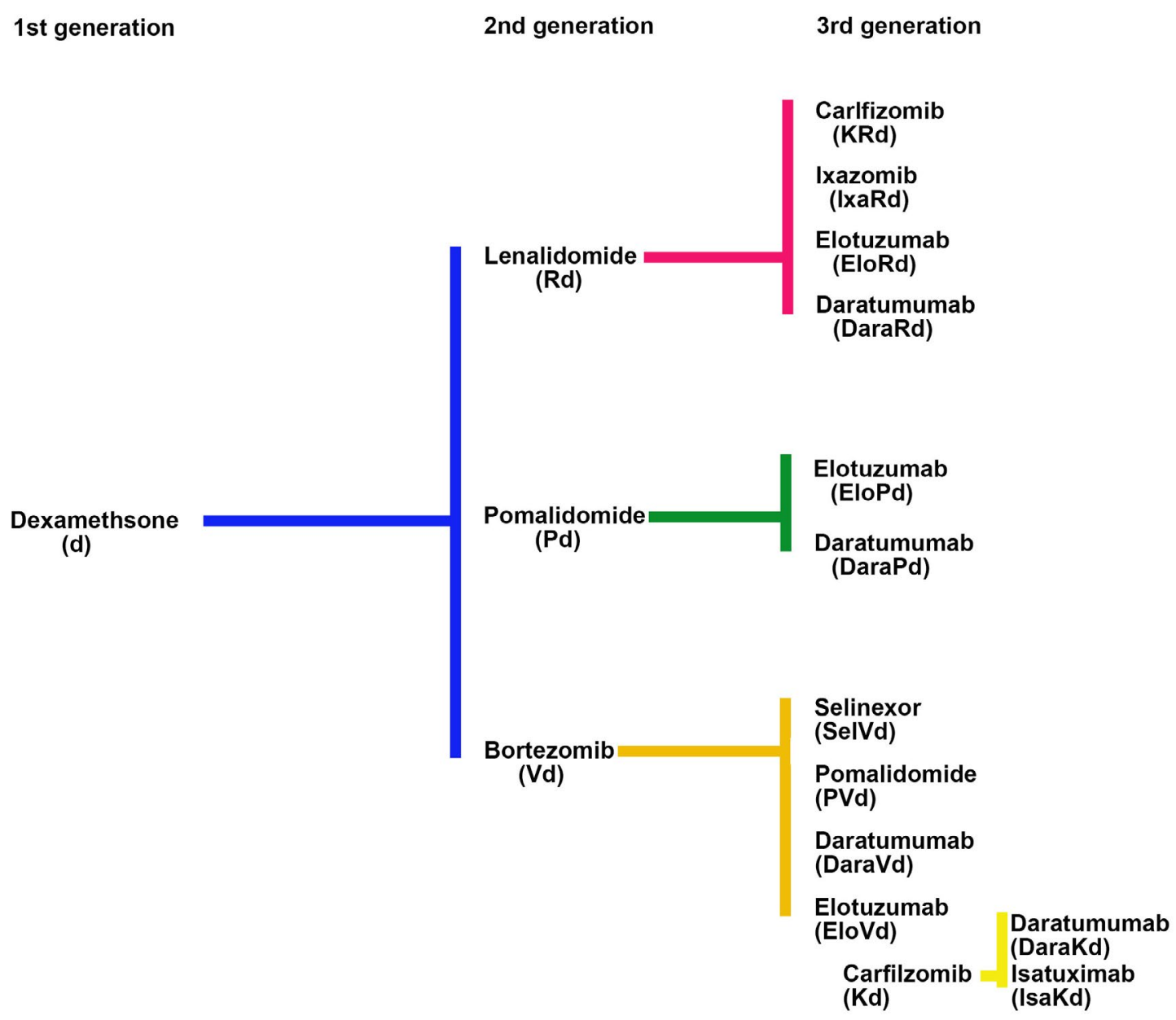

Figure 2. History of comparator arms in relapsed refractory trials (1-3 prior lines)

The immunomodulatory drugs

Thalidomide was developed in the mid-1950s for use as a sedative with minimal side effects or addictive potential [27]. By 1961 it was taken off the market worldwide after teratogenic effects were noted; close to 10,000 infants were thought to have limb deformities caused by the drug. The observation that thalidomide 
resulted in teratogenesis led investigators to consider its effect as an antineoplastic drug, though early efforts failed to show significant activity in advanced cancer [28, 29].

Thalidomide was initially successful as a treatment for erythema nodosum leprosum and inflammatory ulcers. Decreased tumor necrosis factor-alpha and inhibition of vascular endothelial growth factor and fibroblast growth factors were found to be the mechanism for anti-inflammatory and antiangiogenetic properties. In the 1990s, the antiangiogenic properties of thalidomide were discovered [30] and its promise as a cancer therapeutic emerged largely through kismet. A patient's family member noticed the association of thalidomide activity with anti-angiogenesis and, also noted that MM was associated with an excess of neovascular formation. Following a conversation with their doctor a clinical trial in MM was initiated [31]. Later, it was shown that other mechanisms of action of thalidomide included impairment of cell-cell signaling, inhibition of cytokines important to plasma cell growth proliferation, free radical-mediated DNA damage, and enhancement of $\mathrm{T}$ and natural killer (NK) cell responses [32-35]. However, it was years from this time point until the mechanism of action of thalidomide was fully understood, when cereblon, a receptor of the E3 ubiquitin ligase complex, was identified as a thalidomide-binding protein leading to proteasomal degradation of key plasma cell transcription factors Ikaros and Aiolos [31, 36, 37].

Early clinical trials demonstrated responses with single agent thalidomide. Among 84 patients with refractory MM, thalidomide had an overall response rate (ORR) of $32 \%$ with two complete responses [38]. A number of studies compared thalidomide to dexamethasone and evaluated the two drugs in combination in the relapsed setting [39, 40]. As initial therapy, thalidomide in combination with doxorubicin and dexamethasone (TAD) was compared to what was then the European standard induction regimen for transplant eligible patients, VAD [41]. The superiority of TAD had a significant impact on the therapeutic landscape, ultimately leading to the "death of VAD" as a clinically used regimen, the licensing of thalidomide in combination with dexamethasone in 2006, and the development of the Risk Evaluation and Mitigation Strategies (REMS) program [42]. The REMS program is now in widespread use to prevent exposure of "at risk" individuals such as pregnant women to thalidomide, and the principle to decrease exposure has been extended to other anti-cancer drugs that may also be considered at high risk of teratogenicity.

Since the introduction of thalidomide and its derivative lenalidomide, IMiDs have become a critical backbone of standard induction regimens. In the process of introducing these drugs it was shown in relapsed patients that a lenalidomide-dexamethasone combination was more effective when compared to dexamethasone alone [43-45]. A third thalidomide derivative, pomalidomide, was developed in a similar fashion, leveraging off-label use of dexamethasone as its control arm, and has become a key component of treatment for relapsed disease.

Prior to the official approval of IMiDs, they were widely employed in combination with dexamethasone by investigators in clinical trials, leading to greater experience and optimization of their use. IMiDs were initially given in a continuous fashion as maintenance for patients post-HDM with ASCT, and following the combination of melphalan, thalidomide and prednisone for non-transplant candidates before formal approval in these settings. The side effect profile of thalidomide, however, was not ideal for long term maintenance therapy after investigator-initiated trials noted toxicities such as constipation, somnolence, and neuropathy [46] and it was soon replaced by lenalidomide. A number of clinical trials explored lenalidomide as maintenance following HDM and ASCT [47-49] using two different dosing regimens: 10-15 mg continuously and $10 \mathrm{mg}$ for 21 out of 28 days. Its use was associated with myelosuppression that led to discontinuation as maintenance, especially in older patients [49]. Intermittent dosing was associated with less myelosuppression [48], and this became the regimen now widely in use for maintenance, despite lenalidomide not having an FDA label for this dose schedule [50].

The efficacy of combination therapy with IMiDs and dexamethasone in relapsed MM provided a new FDA-approved comparator group for future clinical trials, allowing the field to move from the use of doublet combinations to combinations based on two new agents together with dexamethasone. Each successive improvement was compared to the previous standard of care, all relating back to the initial comparison to dexamethasone used without an FDA label (Figure 2). 


\section{The proteasome inhibitors}

The development of PIs took a parallel and, in some ways, competitive path to the development of the IMiDs. The initial phase II trial that led to the approval of bortezomib [51] evaluated a dose of $1.3 \mathrm{mg} / \mathrm{m}^{2}$ on days 1 , 4, 8 and 11 intravenously. This dosing schedule was associated with significant peripheral neuropathy [52] and a dose reduction was incorporated into future clinical trials to abrogate the toxicity. Despite the side effect profile, the original dose was approved [53], and was the dosing schedule chosen for the drug's subsequent development, for example with melphalan as part of the Velcade-MP (VMP) regimen [54]. As it was the approved dosage all subsequent regulatory trials were compared to this dose and schedule even though clinicians learned to manage the toxicity by using off-label weekly dosing, and rapidly switched to the use of subcutaneous injections when a preparation was available. Therefore, outside of the regulatory setting the dose was reduced to $1.3 \mathrm{mg} / \mathrm{m}^{2}$ subcutaneously at weekly intervals-dosing that has never been formally tested against a control in a randomized trial and is based on small phase II datasets [55-57]. The use of standardized dosages during the approval process is important; however, this need has to be balanced against knowledge gained by real-world drug use obtained post-FDA approval to minimize side effects. In this case the newer dosing frequency decreased the rate of neuropathy and prolonged the length of time a patient was able to remain on drug. Such an approach does cause confusion when subsequent lines of therapy are developed, as ideally the original dosing schedule in the FDA label should be used as a standard against which to compare new therapies.

\section{Developing and utilizing the concept of RRMM}

An important theme of drug development in MM has been the strategy of introducing successive waves of potentially useful clinical drugs for relapsed patients who have received all available therapies-so called "areas of unmet clinical need". As discussed above, the therapies available 5-10 years ago included an IMiD, a PI and possibly an alkylator. In the early 2000s, once a drug was shown to be effective in patients with relapsed or refractory disease, combinations of the new drug with known effective drugs were developed including doublet, triplet and quadruplet combinations. These combinations were then examined in an earlier relapse setting before moving to the newly diagnosed setting.

By 2010-2015 with an increasing number of effective therapies, the need for a different approach with tighter definitions of eligible patients was needed, leading to the concept of relapsed/refractory MM (RRMM) in drug development. This was defined as progressive (within 60 days) or refractory disease during or after the receipt of prior therapy according to strict International Myeloma Working Group (IMWG) criteria [58-60]. Retrospective data analyses showed that as a group RRMM was associated with an extremely poor prognosis. In a cohort of 543 patients who had received a median of four prior lines of therapy, median PFS was 5 months and OS was 13 months [61]. This constituted a well-defined group with an unmet medical need that could provide a path forward for rapid new drug approval. After testing novel drugs alone and in combination in this setting, they could be then moved forward for evaluation in patients with RRMM after 1-3 prior lines of therapy, and subsequently for NDMM.

\section{Anti-CD38 antibodies}

Anti-CD38 monoclonal antibodies have moved rapidly from an experimental drug to standard of care in various MM disease settings [62]. The initial studies of daratumumab showed a 30\% ORR as a single agent in RRMM [63-65]. Since this observation, the drug has undergone extensive clinical trials in the relapsed setting (1-3 lines of therapy) where it has been combined with lenalidomide and dexamethasone (Rd) [66], bortezomib and dexamethasone (Vd) [67], carfilzomib and dexamethasone [68], as well as pomalidomide and dexamethasone $[69,70]$. Isatuximab, another anti-CD38 antibody, is approved for use in combination with pomalidomide and dexamethasone [71] and is undergoing clinical trials with other drug combinations. Similar combinations have recently been approved in the upfront setting including daratumumabVelcade-melphalan-prednisone (D-VMP), daratumumab-Revlimid-dexamethasone (DRd) for transplant ineligible patients, and daratumumab-Velcade-thalidomide-dexamethasone (D-VTD) for transplant eligible patients [72-74]. Compared to Europe where D-VMP, DRd and D-VTD are widely used for NDMM patients, in 
the US, Velcade-Revlimid-dexamethasone (VRD) is the standard of care for both patient groups. Although not yet FDA-approved, results from the Griffin study and extrapolation of other combination data showing good efficacy and safety profiles have led to the widespread use of D-VRD upfront [75]. Of note the FDA approvals for the subcutaneous form of daratumumab compared to the intravenous form also differ slightly [76, 77]. This is again due to the timing differences in the introduction of the two formulations. Even though the data concerning combinations with subcutaneous daratumumab upfront are awaited, there has been widespread uptake of this formulation in clinical practice, driven by the huge difference in infusion time (4-8 $\mathrm{h} v \mathrm{vs} .5 \mathrm{~min})$ and the advantages this has for both patients and clinics.

\section{Developing the concept of penta-refractory disease}

Once anti-CD38 agents became widely available the clinical nature and history of patients with RRMM changed and the old definition of RRMM was no longer appropriate. This resulted in two new functional definitions, which are currently still in use: triple class-exposed (prior IMiD, PI, and anti-CD38 antibody) and penta-refractory (prior exposure and refractoriness to bortezomib, both lenalidomide and pomalidomide, and daratumumab). An updated analysis of RRMM outcomes following the introduction of daratumumab identified a median OS of only 5.6 months for patients considered as triple-exposed and penta-refractory [78]. Again, this represented a new area of unmet medical need where patients continue to have an extremely poor outcome after exposure and refractoriness to available therapies.

Clinical trials are difficult to perform in this area as patients tend to be unwell, and a full prior medical history with access to detailed medical records is required to ensure that patients meet the stringent definitions. It has also become clear that although this is a well-defined group, not all patients have the same biological disease. For example, patients may become penta-refractory over a 10-year period having received each drug sequentially or they may reach the same clinical state quickly over an 18-month period having received two different combinations of therapy. In addition, these definitions do not take into account clinical characteristics such as extramedullary disease or plasma cell leukemia, or genetic features such as mutations or translocations involving MAF, NSD2, MYC, or TP53.

Despite such difficulties, two drugs have been developed in this setting. The first is an antibody-drug conjugate belantamab mafadotin [79] that targets the B-cell maturation antigen (BCMA) which is highly expressed on MM cells [80, 81]. The anti-BCMA monoclonal antibody is conjugated to monomethyl auristatin, a microtubule disrupter resulting in targeted MM cell death. As a single agent in RRMM it showed a 30$34 \%$ ORR depending on the dose used [79]. The second therapy developed in this setting is the nuclear export inhibitor Selinexor [82], which blocks exportin 1, promoting apoptosis of malignant plasma cells by maintaining tumor suppressor proteins within the cell nucleus. In a trial of 122 patients with RRMM who were at least triple-class-exposed, selinexor combined with dexamethasone produced an ORR of $26 \%$ including two stringent complete responses. Given the activity of these therapies, use outside of their label will likely occur, i.e., in patients who fail to meet the strict criteria of penta-refractory disease but who in the physician's eyes are likely to benefit from therapy.

\section{The challenge moving forward with new drug development}

In addition to belantamab mafadotin and selinexor, a number of other drugs with novel mechanisms of action are also being developed in this penta-refractory setting, including T-cell engaging therapy either with chimeric antigen receptor (CAR) T-cells recognizing antigens expressed highly on MM cells or with bi-specific and tri-specific antibodies recognizing MM antigens and T-cells simultaneously.

Other novel drug classes include melphalan flufenamide (melflufen), a peptide-drug conjugate that delivers an alkylating agent directly into MM cells [83-86]. In a phase II trial of heavily pretreated patients, in combination with dexamethasone, melflufen had an ORR of 31\% [87]. In an ongoing phase III trial of patients with RRMM, melflufen plus dexamethasone will be compared to pomalidomide and dexamethasone.

At the moment with only phase I and II data available for these emerging therapies, the temptation is to carry out cross-trial comparison in the penta-refractory setting in order to determine the optimum agent for 
an individual patient. As discussed above this approach is fraught with difficulties and can be misleading due to the composition of small biologically variable groups.

For pharmaceutical companies the challenge moving forward is how to develop a drug in such a crowded setting. There still remains an unmet need as patients continue to relapse, but do patients now need to be refractory to belantamab mafadotin and selinexor in order to receive these new agents? What happens in cases where it is not clinically appropriate that a patient receives all these drugs? Do therapies need to be given in a pre-determined order? For a phase III trial how is an appropriate comparator chosen? Is the "new RRMM" setting actually the most appropriate position to develop new agents? And finally, maybe most importantly, how does individual patient biology determine response to therapy? It will be critical to address these questions going forward for a disease where previously there was only one available therapy (Figure 3).

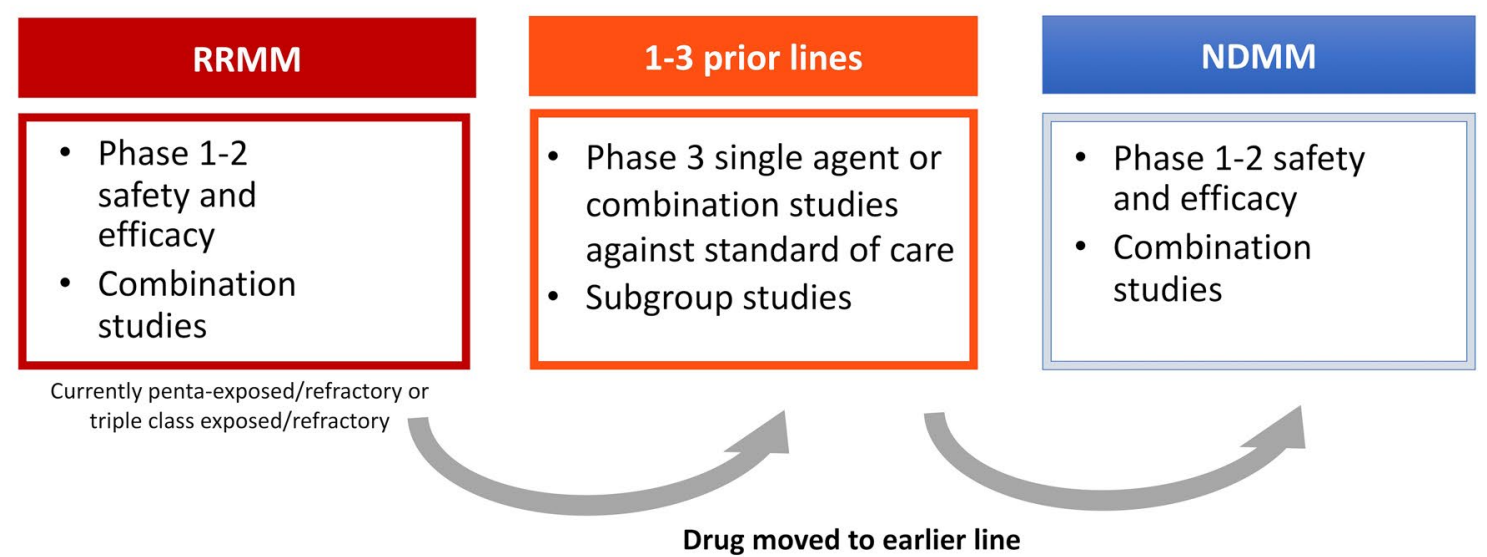

Figure 3. Traditional flow of drug development in $\mathrm{MM}$

\section{The situation in patients not destined for transplantation}

In older less-fit patients with MM who are not candidates for ASCT, drug development and off-label drug use has also occurred. Early studies explored combinations of low dose melphalan with a steroid followed by the addition of a third agent, such as thalidomide, bortezomib or lenalidomide. In this group of patients, it became obvious that combinations had the potential to be detrimental for frail patients, as the side effect profile reduced quality of life and decreased efficacy. The classic example of this was the combination of melphalan and prednisone with lenalidomide, where older patients did not achieve a survival benefit [88]. The FIRST trial established Rd as an alternative to a melphalan combination [89], leading eventually to the MAIA study which demonstrated that lenalidomide plus daratumumab (DRd) outperformed Rd, and importantly was not detrimental to quality of life in this frailer patient population [72]. The countrywide differences in the drug approval processes and marketing authorizations has led to quite dramatic differences in what is considered standard of care for this patient group. For example, in the US, off-label experience with dose-reduced VRD in older populations established the efficacy of this combination. This resulted in a clinical situation where VRD with daratumumab had largely become the standard for all groups, with performance status or frailty score being used to decide on the appropriate combination and dosing, whereas DRd, VMP and D-VMP are often used as standards in other countries.

\section{The impact of the European Union}

For clarity we have focused predominantly on the evolution of therapies in the US, but of course, the European Union (EU), Canada, Asia-Pacific and South America exerted a significant impact on the use of therapies both on and off-label in the individual countries and in the US. Critical features impacting off-label use that differentiates these areas from the US are the different regulatory processes and the factors governing reimbursement in individual countries. For example, the EU regulatory authorities have tended to insist on evidence derived from randomized trials with PFS and OS as end points and have not had a fast track to licensing based on single arm studies in RRMM where response rate and PFS are end points. This has led to different time frames for approvals of new agents, different clinical trial designs to meet the evidence requirements of the regulatory agencies and the important role of country-specific data generated by many 
collaborative groups such as the IFM in France, MRC in the UK, Hovon in Holland, and the GMMG and DSSM in Germany. A key example of the country/regional difference is the use of VRD as a standard in the US, whereas in Europe, VTD and VMP were considered standards. Thus, when data emerged from large randomized studies based in Europe such as D-VTD and D-VMP they had little relevance in the US, and although FDAapproved were not widely utilized.

Perhaps the major factor driving the evolution of different preferred regimens in countries are the reimbursement procedures. With the complexity of drug costs and different healthcare systems, in addition to an assessment of safety and efficacy performed at the regulatory level, there is often an assessment of "value", performed either at an insurance company level or a national level. For example, the European Medical Authority (EMA) may approve a license but the individual countries of the EU will then assess locally how the drug will be reimbursed within their country. The definition and methodologies behind the term "value" varies, as it takes in to account not only the expected benefit in terms of prolonged life for an individual patient, but the impact of this on the wider community (e.g., quality of life, productivity and contribution to the wider community, value in comparison to other health care advancements). It is therefore easy to see that with varying financial situations in the countries and different approval/reimbursement processes that large differences in both on and off-label prescribing can evolve. Ultimately though, the weight of the data and technological advances such as the recent introduction of anti-CD38 antibodies leads to a rebalancing of the system. This is in the process of happening with the global uptake of VRD combined with daratumumab as a global standard.

\section{Use of drugs that modulate novel targets that are labeled for use in another cancer}

With increasing understanding of the genetic and molecular pathogenesis of MM, new potential therapeutic targets have emerged that may be amenable to inhibition by using drugs that have been developed for the same target in another cancer type. MM is characterized by recurrent chromosomal translocations involving the immunoglobulin heavy chain locus on chromosome 14, where genes are brought next to an immunoglobulin enhancer leading to dysregulated gene expression. For example, the $t(4 ; 14)$, present in $15 \%$ of MM $[90,91]$, leads to increased expression of the Wolf-Hirschhorn syndrome candidate gene (WHSC1, also known as MMSET and now NSD2) and the receptor tyrosine kinase fibroblast growth factor receptor 3 (FGFR3) gene $[92,93]$. Inhibition of FGFR3 has been shown to induce plasma cell differentiation and apoptosis in vitro $[94,95]$.

Evaluating novel agents which may have activity in other cancers in MM where specific mutations are identified only in a small percentage of patients requires an "as yet to be determined standardized approach" to avoid further off-label prescriptions. Such an approach will probably involve an umbrella or basket trial design. An example is the ongoing NCI-MATCH study [96], which is "tumor pathogenic-type agnostic," and uses molecularly targeted agents directed against the pathogenic mutation. As part of this trial, which did not include MM patients, AZD4547, an FGFR1-3 tyrosine kinase inhibitor was tested on 48 patients but did not meet its pre-specified efficacy endpoint for ORR.

Increasing knowledge derived from sequencing analysis of MM has provided more potential off-label targets. Mutational analysis of MM identified the central role played by mutations in the rat sarcoma (RAS)/ mitogen activated protein kinase (MAPK) pathway suggesting it is a major target for therapeutic manipulation. Neuroblastoma-RAS (NRAS), Kirsten-RAS (KRAS) and BRAF are mutated in 25\%, 25\%, and 4-8\% of cases respectively in NDMM $[97,98]$. Mutations in these genes are one of the few genomic differences discovered to date between monoclonal gammopathy of undetermined significance (MGUS) and MM, suggesting an important role in the evolution from a precursor state to symptomatic disease. In a retrospective study of 40 patients with heavily pre-treated $\mathrm{MM}$, nine had a complete response to off-label use of trametinib, an allosteric inhibitor of mitogen-activated protein kinase (MEK)1/2 [99]. Successful use of the BRAF inhibitor, vemurafenib, has also been seen, following the initial report in a patient with heavily pretreated MM and 
extramedullary disease [100]. Its use is currently being evaluated in a phase II study with preliminary results showing an $82 \%$ ORR [101].

The ongoing phase I/II Myeloma-Developing Regimens Using Genomics (MyDRUG) trial plans to leverage drugs approved for another indication [102]. MM patients with mutations in CDKN2C, FGFR3, KRAS, NRAS, BRAFV600E, IDH2, or the $\mathrm{t}(11 ; 14)$ translocation are eligible. In combination with ixazomib, pomalidomide, and dexamethasone (IPd), based on their genomic targets enrolled patients will receive the drugs abemaciclib, enasidenib, cobimetinib, erdafitinib, or venetoclax. Patients without one of the targetable mutations described above will receive daratumumab in combination with IPd. This is not a registration trial for approval of these drugs for MM, but rather an example of testing therapies developed in other malignancies for use off-label.

\section{Can the story of thalidomide be recapitulated by repurposing other drugs for use in MM?}

Given the poor prognosis of RRMM and the difficulty of approving novel drugs there is great interest in maximizing the use of a drug in more than one cancer indication as well as repurposing drugs already approved for another disease indication. The FDA ensures drugs are safe and effective, based on the Food, Drug, and Cosmetic Act of 1938 and the Kefauver-Harris Amendment, respectively [103]. Once approved for one indication, physicians are not prohibited from prescribing for indications or in doses not specified in the initial approval. The formal approval process for new drugs is long and costly. New drug approval is estimated to take 10-20 years [104] and cost up to $\$ 2$ billion [105]. The large majority of these drugs fail during clinical development: between 2003 and 2011 an estimated 6.7\% of drugs tested in a phase I trial for an oncologic indication made it to FDA approval [106]. At the same time, the cost of new cancer therapies is rising, contributing to increasing financial costs for both individual patients and to healthcare systems [107, 108].

Considering the cost and difficulty of developing novel therapies, one strategy has been the repurposing of drugs already available for a different disease. Through programs such as the ReDO project [109] there is ongoing work to repurpose existing low-cost drugs. The anti-helmenthinc mebendazole, for example, has been shown in vitro and in mouse models to inhibit tumorogenesis in lung cancer [110, 111], adrenal tumors [112], melanoma [113], glioblastoma multiforme [114], and breast cancer [115]. In MM, mebendazole delayed tumor growth in mice, possibly through the USP5/c-Maf pathway [116]. Nocodazole, another anti-helmenthic, inhibited tumor growth via cell cycle arrest and microtubule dysfunction, associated with increased Bim and myeloid cell leukemia-1 (MCL-1) expression and c-Jun NH2-terminal kinase (JNK)mediated B cell lymphoma protein-2 (BCL-2) phosphorylation [117]. Despite these promising in vitro results it appears the introduction of these agents into the clinic is unlikely, but the examples demonstrate the possibilities of such an approach.

Another example is the antibiotic clarithromycin, which shows high response rates when combined with $\mathrm{Rd}$ [118], and had a higher response rate than Rd alone when compared retrospectively [119]. A phase II trial evaluated clarithromycin combined with pomalidomide and dexamethasone and showed a $60 \%$ ORR [120]. Proposed mechanisms for clarithromycin's effect include increasing the concentration of dexamethasone through cytochrome P450 3A4 (CYP3A4) inhibition, downregulation of T-reg cell response via decreased interleukin (IL)-6 and increased IL-10 and interferon (IFN)-y levels [121, 122], and through attenuation of autophagy in MM cells [123]. Despite the enthusiasm concerning the initial results the combination has not been widely taken up because of uncertainty about the mechanism or its clinical relevance.

The combination of the protease inhibitor ritonavir with metformin has shown antitumor activity in MM both in vitro and in vivo with xenograft mouse models, through inhibition of glucose transporter 4 (GLUT4) and mitochondrial oxidative metabolism, leading to suppression of signaling through the protein kinase B (AKT)/ mammalian target of rapamycin complex 1 (mTORC1) pathway known to regulate MCL-1 [124]. Ritonavir has also been shown to sensitize MM cells to the effect of bortezomib [125]. Early trial data showed response rates to nelfinavir, another protease inhibitor, in patients who were previously exposed and resistant to bortezomib $[126,127]$. This approach has not been widely taken up because of the subsequent development 
of agents targeting BCMA, however the clinical experience with the combination was that it could be useful and was not toxic, making these agents potential alternatives if recently approved agents were not available.

A further example is venetoclax, a BCL-2 inhibitor that is approved for use in chronic lymphocytic leukemia (CLL) and acute myelogenous leukemia (AML). Early clinical trials in RRMM, as well as case reports in plasma cell leukemia and amyloid disease show it is particularly effective in patients with a $\mathrm{t}(11 ; 14)$ [128-130]. Its development in MM has been checkered as the early studies investigated all MM patients, not just $\mathrm{t}(11 ; 14)$ cases, and although it was found to be more efficacious in combination with $\mathrm{Vd}$ compared to Vd alone, it was also noted to have increased toxicity leading to a pause in development [131]. Studies have now re-commenced in the group of patients where retrospective case reports had shown unprecedented activity — those with $\mathrm{t}(11 ; 14)$ —and it is hoped the drug will eventually gain formal approval in this space.

\section{Conclusions}

In the earlier era of alkylating agents and corticosteroids, off-label drug use filled a void in available therapies and was critical to the improved outcomes seen in MM today. With the advances in the number of active MM therapies, the clinical trial and regulatory approval framework has continually evolved to accommodate the safe and effective investigation and approval of new therapies. Moving forward we firmly believe that patients wherever possible should be treated within the context of clinical trials, that off-label use should be kept to a minimum, and safety and efficacy data reported. We do however recognize that off-label use continues in MM therapy. Currently, a frequent and notable off-label use is during development of novel therapies where use of a therapy becomes widespread following the release of promising clinical trial results prior to regulatory approval. With further understanding of the molecular and cellular mechanisms of MM and cancer pathogenesis generally there has been a move toward targeted therapies being used across multiple cancer types. This has led to a further off-label area for "niche therapies" for small well-defined patient populations where large clinical trials with sufficient power may be difficult to perform. Finally, we have seen that drugs initially used for non-neoplastic indications have activity in MM, leading to the possibility of cheap and safe drugs already in production becoming effective components of combination therapy. Cautionary use of off-label prescribing has provided and will continue to provide a vehicle to enhance the treatment of MM patients with the introduction of effective treatment regimens in a disease setting where prognosis is poor and there remains an unmet medical need.

\section{Abbreviations}

ASCT: autologous stem cell transplantation

BCMA: B-cell maturation antigen

DRd: daratumumab-Revlimid-dexamethasone

D-VMP: daratumumab-Velcade-melphalan-prednisone

D-VTD: daratumumab-Velcade-thalidomide-dexamethasone

EU: European Union

FDA: Food and Drug Administration

FGFR3: fibroblast growth factor receptor 3

HDM: high-dose melphalan

IMiD: immunomodulatory drug

MM: multiple myeloma

MP: melphalan-prednisone

NDMM: newly diagnosed multiple myeloma

ORR: overall response rate

OS: overall survival 
PFS: progression free survival

PI: proteasome inhibitor

Rd: Revlimid-dexamethasone

RRMM: relapsed/refractory multiple myeloma

VAD: vincristine-doxorubicin-dexamethasone

Vd: Velcade-dexamethasone

VMP: Velcade-melphalan-prednisone

VRD: Velcade-Revlimid-dexamethasone

VTD-PACE: Velcade-thalidomide-dexamethasone-cisplatin-doxorubicin-cyclophosphamide-etoposide

\section{Declarations}

\section{Author contributions}

JHS, FED, EMB, LW and GJM wrote the manuscript. All authors contributed to manuscript revisions and read and approved the submitted version.

\section{Conflicts of interest}

JHS, EMB and LW declare that they have no conflicts of interest. FED has participated in advisory boards for BMS, GSK, Janssen, Oncopeptides, Roche, Takeda, and has consulted for Adaptive, BMS, Janssen, and Sanofi. GJM has participated in advisory boards for BMS, GSK, Janssen, Oncopeptides, and Roche.

\section{Ethical approval}

Not applicable.

\section{Consent to participate}

Not applicable.

\section{Consent to publication}

Not applicable.

\section{Availability of data and materials}

Not applicable.

\section{Funding}

Not applicable.

\section{Copyright}

(c) The Author(s) 2021.

\section{References}

1. Drugs approved for multiple myeloma and other plasma cell neoplasms [Internet]. Bethesda: NIH; [updated 2021 Jan 14; cited 2021 Jan 15]. Available from: https://www.cancer.gov/about-cancer/ treatment/drugs/multiple-myeloma

2. Radley DC, Finkelstein SN, Stafford RS. Off-label prescribing among office-based physicians. Arch Intern Med. 2006;166:1021-6.

3. Alexander GC, Gallagher SA, Mascola A, Moloney RM, Stafford RS. Increasing off-label use of antipsychotic medications in the United States, 1995-2008. Pharmacoepidemiol Drug Saf. 2011;20:177-84.

4. Lat I, Micek S, Janzen J, Cohen H, Olsen K, Haas C. Off-label medication use in adult critical care patients. J Crit Care. 2011;26:89-94. 
5. Loder EW, Biondi DM. Off-label prescribing of drugs in specialty headache practice. Headache. 2004;44:636-41.

6. McElwain TJ, Powles RL. High-dose intravenous melphalan for plasma-cell leukaemia and myeloma. Lancet. 1983;2:822-4.

7. Barlogie B, Hall R, Zander A, Dicke K, Alexanian R. High-dose melphalan with autologous bone marrow transplantation for multiple myeloma. Blood. 1986;67:1298-301.

8. Barlogie B, Alexanian R, Dicke KA, Zagars G, Spitzer G, Jagannath S, et al. High-dose chemoradiotherapy and autologous bone marrow transplantation for resistant multiple myeloma. Blood. 1987;70:869-72.

9. Attal M, Harousseau JL, Stoppa AM, Sotto JJ, Fuzibet JG, Rossi JF, et al. A prospective, randomized trial of autologous bone marrow transplantation and chemotherapy in multiple myeloma. Intergroupe Francais du Myelome. N Engl J Med. 1996;335:91-7.

10. Child JA, Morgan GJ, Davies FE, Owen RG, Bell SE, Hawkins K, et al. High-dose chemotherapy with hematopoietic stem-cell rescue for multiple myeloma. N Engl J Med. 2003;348:1875-83.

11. Fermand JP, Katsahian S, Divine M, Leblond V, Dreyfus F, Macro M, et al. High-dose therapy and autologous blood stem-cell transplantation compared with conventional treatment in myeloma patients aged 55 to 65 years: long-term results of a randomized control trial from the Group Myelome-Autogreffe. J Clin Oncol. 2005;23:9227-33.

12. Barlogie B, Kyle RA, Anderson KC, Greipp PR, Lazarus HM, Hurd DD, et al. Standard chemotherapy compared with high-dose chemoradiotherapy for multiple myeloma: final results of phase III US Intergroup Trial S9321. J Clin Oncol. 2006;24:929-36.

13. Blade J, Rosinol L, Sureda A, Ribera JM, Diaz-Mediavilla J, Garcia-Larana J, et al. High-dose therapy intensification compared with continued standard chemotherapy in multiple myeloma patients responding to the initial chemotherapy: long-term results from a prospective randomized trial from the Spanish cooperative group PETHEMA. Blood. 2005;106:3755-9.

14. Kumar S, Flinn I, Richardson PG, Hari P, Callander N, Noga SJ, et al. Randomized, multicenter, phase 2 study (EVOLUTION) of combinations of bortezomib, dexamethasone, cyclophosphamide, and lenalidomide in previously untreated multiple myeloma. Blood. 2012;119:4375-82.

15. Jackson GH, Davies FE, Pawlyn C, Cairns DA, Striha A, Collett C, et al. Response-adapted intensification with cyclophosphamide, bortezomib, and dexamethasone versus no intensification in patients with newly diagnosed multiple myeloma (Myeloma XI): a multicentre, open-label, randomised, phase 3 trial. Lancet Haematol. 2019;6:e616-29.

16. Alexanian R, Dimopoulos MA, Delasalle K, Barlogie B. Primary dexamethasone treatment of multiple myeloma. Blood. 1992;80:887-90.

17. FDA. Dexamethasoneprescribing information. Available from: https://www.accessdata.fda.gov/ drugsatfda_docs/label/2019/211379s000lbl.pdf

18. Kumar S, Lacy MQ Dispenzieri A, Rajkumar SV, Fonseca R, Geyer S, et al. Single agent dexamethasone for pre-stem cell transplant induction therapy for multiple myeloma. Bone Marrow Transplant. 2004;34:485-90.

19. Burwick N, Sharma S. Glucocorticoids in multiple myeloma: past, present, and future. Ann Hematol. 2019;98:19-28.

20. Combination chemotherapy versus melphalan plus prednisone as treatment for multiple myeloma: an overview of 6,633 patients from 27 randomized trials. Myeloma Trialists' Collaborative Group. J Clin Oncol. 1998;16:3832-42.

21. Barlogie B, Smith L, Alexanian R. Effective treatment of advanced multiple myeloma refractory to alkylating agents. N Engl J Med. 1984;310:1353-6.

22. Alexanian R, Barlogie B, Dixon D. High-dose glucocorticoid treatment of resistant myeloma. Ann Intern Med. 1986;105:8-11. 
23. Rajkumar SV, Jacobus S, Callander NS, Fonseca R, Vesole DH, Williams ME, et al. Lenalidomide plus highdose dexamethasone versus lenalidomide plus low-dose dexamethasone as initial therapy for newly diagnosed multiple myeloma: an open-label randomised controlled trial. Lancet Oncol. 2010;11:29-37.

24. Orlowski RZ, Nagler A, Sonneveld P, Bladé J, Hajek R, Spencer A, et al. Randomized phase III study of pegylated liposomal doxorubicin plus bortezomib compared with bortezomib alone in relapsed or refractory multiple myeloma: combination therapy improves time to progression. J Clin Oncol. 2007;25:3892-901.

25. Lee CK, Barlogie B, Munshi N, Zangari M, Fassas A, Jacobson J, et al. DTPACE: an effective, novel combination chemotherapy with thalidomide for previously treated patients with myeloma. J Clin Oncol. 2003;21:2732-9.

26. Nishimura KK, Barlogie B, van Rhee F, Zangari M, Walker BA, Rosenthal A, et al. Long-term outcomes after autologous stem cell transplantation for multiple myeloma. Blood Adv. 2020;4:422-31.

27. Rajkumar SV. Thalidomide: tragic past and promising future. Mayo Clin Proc. 2004;79:899-903.

28. Grabstald H, Golbey R. Clinical experiences with thalidomide in patients with cancer. Clin Pharmacol Ther. 1965;6:298-302.

29. Olson KB, Hall TC, Horton J, Khung CL, Hosley HF. Thalidomide (N-phthaloylglutamimide) in the treatment of advanced cancer. Clin Pharmacol Ther. 1965;6:292-7.

30. D’Amato RJ, Loughnan MS, Flynn E, Folkman J. Thalidomide is an inhibitor of angiogenesis. Proc Natl Acad Sci U S A. 1994;91:4082-5.

31. Lu G, Middleton RE, Sun H, Naniong M, Ott CJ, Mitsiades CS, et al. The myeloma drug lenalidomide promotes the cereblon-dependent destruction of Ikaros proteins. Science. 2014;343:305-9.

32. Davies FE, Raje N, Hideshima T, Lentzsch S, Young G, Tai YT, et al. Thalidomide and immunomodulatory derivatives augment natural killer cell cytotoxicity in multiple myeloma. Blood. 2001;98:210-6.

33. Hideshima T, Chauhan D, Shima Y, Raje N, Davies FE, Tai YT, et al. Thalidomide and its analogs overcome drug resistance of human multiple myeloma cells to conventional therapy. Blood. 2000;96:2943-50.

34. Lentzsch S, LeBlanc R, Podar K, Davies F, Lin B, Hideshima T, et al. Immunomodulatory analogs of thalidomide inhibit growth of Hs Sultan cells and angiogenesis in vivo. Leukemia. 2003;17:41-4.

35. Mitsiades N, Mitsiades CS, Poulaki V, Chauhan D, Richardson PG, Hideshima T, et al. Apoptotic signaling induced by immunomodulatory thalidomide analogs in human multiple myeloma cells: therapeutic implications. Blood. 2002;99:4525-30.

36. Ito T, Ando H, Handa H. Teratogenic effects of thalidomide: molecular mechanisms. Cell Mol Life Sci. 2011;68:1569-79.

37. Krönke J, Udeshi ND, Narla A, Grauman P, Hurst SN, McConkey M, et al. Lenalidomide causes selective degradation of IKZF1 and IKZF3 in multiple myeloma cells. Science. 2014;343:301-5.

38. Singhal S, Mehta J, Desikan R, Ayers D, Roberson P, Eddlemon P, et al. Antitumor activity of thalidomide in refractory multiple myeloma. N Engl J Med. 1999;341:1565-71.

39. Dimopoulos MA, Zervas K, Kouvatseas G, Galani E, Grigoraki V, Kiamouris C, et al. Thalidomide and dexamethasone combination for refractory multiple myeloma. Ann Oncol. 2001;12:991-5.

40. Weber D, Rankin K, Gavino M, Delasalle K, Alexanian R. Thalidomide alone or with dexamethasone for previously untreated multiple myeloma. J Clin Oncol. 2003;21:16-9.

41. Cavo M, Zamagni E, Tosi P, Tacchetti P, Cellini C, Cangini D, et al. Superiority of thalidomide and dexamethasone over vincristine-doxorubicindexamethasone (VAD) as primary therapy in preparation for autologous transplantation for multiple myeloma. Blood. 2005;106:35-9.

42. Risk evaluation and mitigation strategies [Internet]. FDA; [updated 2019 Aug 8; cited 2021 Jan 15]. Available from: https://www.fda.gov/drugs/drug-safety-and-availability/risk-evaluation-andmitigation-strategies-rems 
43. Weber DM, Chen C, Niesvizky R, Wang M, Belch A, Stadtmauer EA, et al. Lenalidomide plus dexamethasone for relapsed multiple myeloma in North America. N Engl J Med. 2007;357:2133-42.

44. Dimopoulos M, Spencer A, Attal M, Prince HM, Harousseau JL, Dmoszynska A, et al. Lenalidomide plus dexamethasone for relapsed or refractory multiple myeloma. N Engl J Med. 2007;357:2123-32.

45. Rajkumar SV, Hayman SR, Lacy MQ, Dispenzieri A, Geyer SM, Kabat B, et al. Combination therapy with lenalidomide plus dexamethasone (Rev/Dex) for newly diagnosed myeloma. Blood. 2005;106:4050-3.

46. Morgan GJ, Gregory WM, Davies FE, Bell SE, Szubert AJ, Brown JM, etal. The role of maintenance thalidomide therapy in multiple myeloma: MRC Myeloma IX results and meta-analysis. Blood. 2012;119:7-15.

47. McCarthy PL, Owzar K, Hofmeister CC, Hurd DD, Hassoun H, Richardson PG, et al. Lenalidomide after stem-cell transplantation for multiple myeloma. N Engl J Med. 2012;366:1770-81.

48. Jackson GH, Davies FE, Pawlyn C, Cairns DA, Striha A, Collett C, et al. Lenalidomide maintenance versus observation for patients with newly diagnosed multiple myeloma (Myeloma XI): a multicentre, openlabel, randomised, phase 3 trial. Lancet Oncol. 2019;20:57-73.

49. Attal M, Lauwers-Cances V, Marit G, Caillot D, Moreau P, Facon T, et al. Lenalidomide maintenance after stem-cell transplantation for multiple myeloma. N Engl J Med. 2012;366:1782-91.

50. FDA. Lenalidomide prescribing information. Available from: https://packageinserts.bms.com/pi/pi_ revlimid.pdf

51. Richardson PG, Barlogie B, Berenson J, Singhal S, Jagannath S, Irwin D, et al. A phase 2 study of bortezomib in relapsed, refractory myeloma. N Engl J Med. 2003;348:2609-17.

52. Richardson PG, Sonneveld P, Schuster MW, Irwin D, Stadtmauer EA, Facon T, et al. Bortezomib or highdose dexamethasone for relapsed multiple myeloma. N Engl J Med. 2005;352:2487-98.

53. FDA. Bortezomib prescribing information. Available from: https://www.velcade.com/files/pdfs/ VELCADE_PRESCRIBING_INFORMATION.pdf

54. San Miguel JF, Schlag R, Khuageva NK, Dimopoulos MA, Shpilberg O, Kropff M, et al. Bortezomib plus melphalan and prednisone for initial treatment of multiple myeloma. N Engl J Med. 2008;359:906-17.

55. Moreau P, Mateos MV, Berenson JR, Weisel K, Lazzaro A, Song K, et al. Once weekly versus twice weekly carfilzomib dosing in patients with relapsed and refractory multiple myeloma (A.R.R.O.W.): interim analysis results of a randomised, phase 3 study. Lancet Oncol. 2018;19:953-64.

56. O'Donnell EK, Laubach JP, Yee AJ, Chen T, Huff CA, Basile FG, et al. A phase 2 study of modified lenalidomide, bortezomib and dexamethasone in transplant-ineligible multiple myeloma. Br J Haematol. 2018;182:222-30.

57. Mateos MV, Oriol A, Martínez-López J, Gutiérrez N, Teruel AI, de Paz R, et al. Bortezomib, melphalan, and prednisone versus bortezomib, thalidomide, and prednisone as induction therapy followed by maintenance treatment with bortezomib and thalidomide versus bortezomib and prednisone in elderly patients with untreated multiple myeloma: a randomised trial. Lancet Oncol. 2010;11:934-41.

58. Rajkumar SV, Harousseau JL, Durie B, Anderson KC, Dimopoulos M, Kyle R, et al. Consensus recommendations for the uniform reporting of clinical trials: report of the International Myeloma Workshop Consensus Panel 1. Blood. 2011;117:4691-5.

59. Kumar S, Paiva B, Anderson KC, Durie B, Landgren O, Moreau P, et al. International Myeloma Working Group consensus criteria for response and minimal residual disease assessment in multiple myeloma. Lancet Oncol. 2016;17:e328-46.

60. Durie BG, Harousseau JL, Miguel JS, Blade J, Barlogie B, Anderson K, et al. International uniform response criteria for multiple myeloma. Leukemia. 2006;20:1467-73.

61. Kumar SK, Dimopoulos MA, Kastritis E, Terpos E, Nahi H, Goldschmidt H, et al. Natural history of relapsed myeloma, refractory to immunomodulatory drugs and proteasome inhibitors: a multicenter IMWG study. Leukemia. 2017;31:2443-8. 
62. Network NCC [Internet]. Multiple myeloma (version 4.2021). [updated 2020 Dec 10; cited 2021 Jan 18]. Available from: https://www.nccn.org/professionals/physician_gls/pdf/myeloma.pdf

63. Lokhorst HM, Plesner T, Laubach JP, Nahi H, Gimsing P, Hansson M, et al. Targeting CD38 with daratumumab monotherapy in multiple myeloma. N Engl J Med. 2015;373:1207-19.

64. Lonial S, Weiss BM, Usmani SZ, Singhal S, Chari A, Bahlis NJ, et al. Daratumumab monotherapy in patients with treatment-refractory multiple myeloma (SIRIUS): an open-label, randomised, phase 2 trial. Lancet. 2016;387:1551-60.

65. Usmani SZ, Nahi H, Plesner T, Weiss BM, Bahlis NJ, Belch A, et al. Daratumumab monotherapy in patients with heavily pretreated relapsed or refractory multiple myeloma: final results from the phase 2 GEN501 and SIRIUS trials. Lancet Haematol. 2020;7:e447-55.

66. Dimopoulos MA, Oriol A, Nahi H, San-Miguel J, Bahlis NJ, Usmani SZ, et al. Daratumumab, lenalidomide, and dexamethasone for multiple myeloma. N Engl J Med. 2016;375:1319-31.

67. Palumbo A, Chanan-Khan A, Weisel K, Nooka AK, Masszi T, Beksac M, et al. Daratumumab, bortezomib, and dexamethasone for multiple myeloma. N Engl J Med. 2016;375:754-66.

68. Dimopoulos M, Quach H, Mateos MV, Landgren O, Leleu X, Siegel D, et al. Carfilzomib, dexamethasone, and daratumumab versus carfilzomib and dexamethasone for patients with relapsed or refractory multiple myeloma (CANDOR): results from a randomised, multicentre, open-label, phase 3 study. Lancet. 2020;396:186-97.

69. Siegel DS, Schiller GJ, Samaras C, Sebag M, Berdeja J, Ganguly S, et al. Pomalidomide, dexamethasone, and daratumumab in relapsed refractory multiple myeloma after lenalidomide treatment. Leukemia. 2020;34:3286-97.

70. Chari A, Suvannasankha A, Fay JW, Arnulf B, Kaufman JL, Ifthikharuddin JJ, et al. Daratumumab plus pomalidomide and dexamethasone in relapsed and/or refractory multiple myeloma. Blood. 2017;130:974-81.

71. Attal M, Richardson PG, Rajkumar SV, San-Miguel J, Beksac M, Spicka I, et al. Isatuximab plus pomalidomide and low-dose dexamethasone versus pomalidomide and low-dose dexamethasone in patients with relapsed and refractory multiple myeloma (ICARIA-MM): a randomised, multicentre, open-label, phase 3 study. Lancet. 2019;394:2096-107.

72. Facon T, Kumar S, Plesner T, Orlowski RZ, Moreau P, Bahlis N, et al. Daratumumab plus lenalidomide and dexamethasone for untreated myeloma. N Engl J Med. 2019;380:2104-15.

73. Moreau P, Attal M, Hulin C, Arnulf B, Belhadj K, Benboubker L, et al. Bortezomib, thalidomide, and dexamethasone with or without daratumumab before and after autologous stem-cell transplantation for newly diagnosed multiple myeloma (CASSIOPEIA): a randomised, open-label, phase 3 study. Lancet. 2019;394:29-38.

74. Mateos MV, Cavo M, Blade J, Dimopoulos MA, Suzuki K, Jakubowiak A, et al. Overall survival with daratumumab, bortezomib, melphalan, and prednisone in newly diagnosed multiple myeloma (ALCYONE): a randomised, open-label, phase 3 trial. Lancet. 2020;395:132-41.

75. Voorhees PM, Kaufman JL, Laubach J, Sborov DW, Reeves B, Rodriguez C, et al. Daratumumab, lenalidomide, bortezomib, and dexamethasone for transplant-eligible newly diagnosed multiple myeloma: the GRIFFIN trial. Blood. 2020;136:936-45.

76. FDA. DARZALEX prescribing information. Available from: https://www.janssenlabels.com/packageinsert/product-monograph/prescribing-information/DARZALEX-pi.pdf

77. FDA. DARZALEX FASPRO prescribing information. Available from: https://www.janssenlabels.com/ package-insert/product-monograph/prescribing-information/DARZALEX+Faspro-pi.pdf

78. Gandhi UH, Cornell RF, Lakshman A, Gahvari ZJ, McGehee E, Jagosky MH, et al. Outcomes of patients with multiple myeloma refractory to CD38-targeted monoclonal antibody therapy. Leukemia. 2019;33:2266-75. 
79. Lonial S, Lee HC, Badros A, Trudel S, Nooka AK, Chari A, et al. Belantamab mafodotin for relapsed or refractory multiple myeloma (DREAMM-2): a two-arm, randomised, open-label, phase 2 study. Lancet Oncol. 2020;21:207-21.

80. Carpenter RO, Evbuomwan MO, Pittaluga S, Rose JJ, Raffeld M, Yang S, et al. B-cell maturation antigen is a promising target for adoptive T-cell therapy of multiple myeloma. Clin Cancer Res. 2013;19:2048-60.

81. Novak AJ, Darce JR, Arendt BK, Harder B, Henderson K, Kindsvogel W, et al. Expression of BCMA, TACI, and BAFF-R in multiple myeloma: a mechanism for growth and survival. Blood. 2004;103:689-94.

82. Chari A, Vogl DT, Gavriatopoulou M, Nooka AK, Yee AJ, Huff CA, et al. Oral selinexor-dexamethasone for triple-class refractory multiple myeloma. N Engl J Med. 2019;381:727-38.

83. Chauhan D, Ray A, Viktorsson K, Spira J, Paba-Prada C, Munshi N, et al. In vitro and in vivo antitumor activity of a novel alkylating agent, melphalan-flufenamide, against multiple myeloma cells. Clin Cancer Res. 2013;19:3019-31.

84. Strese S, Wickstrom M, Fuchs PF, Fryknas M, Gerwins P, Dale T, et al. The novel alkylating prodrug melflufen (J1) inhibits angiogenesis in vitro and in vivo. Biochem Pharmacol. 2013;86:888-95.

85. Wickstrom M, Nygren P, Larsson R, Harmenberg J, Lindberg J, Sjoberg P, et al. Melflufen-a peptidasepotentiated alkylating agent in clinical trials. Oncotarget. 2017;8:66641-55.

86. Ray A, Ravillah D, Das DS, Song Y, Nordstrom E, Gullbo J, et al. A novel alkylating agent Melflufen induces irreversible DNA damage and cytotoxicity in multiple myeloma cells. Br J Haematol. 2016;174:397-409.

87. Richardson PG, Bringhen S, Voorhees P, Plesner T, Mellqvist UH, Reeves B, et al. Melflufen plus dexamethasone in relapsed and refractory multiple myeloma (0-12-M1): a multicentre, international, open-label, phase 1-2 study. Lancet Haematol. 2020;7:e395-407.

88. Zweegman S, van der Holt B, Mellqvist UH, Salomo M, Bos GM, Levin MD, et al. Melphalan, prednisone, and lenalidomide versus melphalan, prednisone, and thalidomide in untreated multiple myeloma. Blood. 2016;127:1109-16.

89. Facon T, Dimopoulos MA, Dispenzieri A, Catalano JV, Belch A, Cavo M, et al. Final analysis of survival outcomes in the phase 3 FIRST trial of up-front treatment for multiple myeloma. Blood. 2018;131:301-10.

90. Chesi M, Bergsagel PL, Brents LA, Smith CM, Gerhard DS, Kuehl WM. Dysregulation of cyclin D1 by translocation into an IgH gamma switch region in two multiple myeloma cell lines. Blood. 1996; 88:674-81.

91. Gabrea A, Bergsagel PL, Chesi M, Shou Y, Kuehl WM. Insertion of excised IgH switch sequences causes overexpression of cyclin D1 in a myeloma tumor cell. Mol Cell. 1999;3:119-23.

92. Chesi M, Nardini E, Brents LA, Schrock E, Ried T, Kuehl WM, et al. Frequent translocation t(4;14) (p16.3;q32.3) in multiple myeloma is associated with increased expression and activating mutations of fibroblast growth factor receptor 3. Nat Genet. 1997;16:260-4.

93. Chesi M, Nardini E, Lim RS, Smith KD, Kuehl WM, Bergsagel PL. The t(4;14) translocation in myeloma dysregulates both FGFR3 and a novel gene, MMSET, resulting in IgH/MMSET hybrid transcripts. Blood. 1998;92:3025-34.

94. Trudel S, Ely S, Farooqi Y, Affer M, Robbiani DF, Chesi M, et al. Inhibition of fibroblast growth factor receptor 3 induces differentiation and apoptosis in $\mathrm{t}(4 ; 14)$ myeloma. Blood. 2004;103:3521-8.

95. Trudel S, Stewart AK, Rom E, Wei E, Li ZH, Kotzer S, et al. The inhibitory anti-FGFR3 antibody, PRO-001, is cytotoxic to $\mathrm{t}(4 ; 14)$ multiple myeloma cells. Blood. 2006;107:4039-46.

96. Clinicaltrials.gov [Internet]. The MATCH Screening Trial. [updated 2021 Jan 15; cited Feburary 1, 2021]. Available from: https://www.clinicaltrials.gov/ct2/show/NCT02465060

97. Bezieau S, Devilder MC, Avet-Loiseau H, Mellerin MP, Puthier D, Pennarun E, et al. High incidence of N and K-Ras activating mutations in multiple myeloma and primary plasma cell leukemia at diagnosis. Hum Mutat. 2001;18:212-24. 
98. Walker BA, Boyle EM, Wardell CP, Murison A, Begum DB, Dahir NM, et al. Mutational spectrum, copy number changes, and outcome: results of a sequencing study of patients with newly diagnosed myeloma. J Clin Oncol. 2015;33:3911-20.

99. Heuck CJ, Jethava Y, Khan R, van Rhee F, Zangari M, Chavan S, et al. Inhibiting MEK in MAPK pathwayactivated myeloma. Leukemia. 2016;30:976-80.

100. Andrulis M, Lehners N, Capper D, Penzel R, Heining C, Huellein J, et al. Targeting the BRAF V600E mutation in multiple myeloma. Cancer Discov. 2013;3:862-9.

101. Raab MSea, GiesenN, Scheid C, Besemer B, Miah K, Benner A, et al. 294 safety and preliminary efficacy results from a phase II study evaluating combined BRAF and MEK inhibition in relapsed/refractory multiple myeloma (rrMM) patients with activating BRAF V600E mutations: the GMMG-Birma Trial. Proceedings of the 62nd ASH Annual Meeting and Exposition; 2020 Dec 5-8; Washington. US: ASH; 2020.

102. Auclair D, Anderson KC, Avigan D, Bianchi G, Biran N, Chaudhry M, et al. The myeloma-developing regimens using genomics (MyDRUG) master protocol. J Clin Oncolo. 2019;37:TPS8057.

103. Milestones in U.S. Food and Drug Law History [Internet]. FDA; [updated 2018 Jan 31; cited 2021 Jan 18]. Available from: https://www.fda.gov/about-fda/fdas-evolving-regulatory-powers/milestones-us-foodand-drug-law-history

104. Tobinick EL. The value of drug repositioning in the current pharmaceutical market. Drug News Perspect. 2009;22:119-25.

105. Adams CP, Brantner VV. Estimating the cost of new drug development: is it really 802 million dollars? Health Aff (Millwood). 2006;25:420-8.

106. Hay M, Thomas DW, Craighead JL, Economides C, Rosenthal J. Clinical development success rates for investigational drugs. Nat Biotechnol. 2014;32:40-51.

107. Desai A, Gyawali B. Financial toxicity of cancer treatment: moving the discussion from acknowledgement of the problem to identifying solutions. EClinicalMedicine. 2020;20:100269.

108. Ekwueme DU, Zhao J, Rim SH, de Moor JS, Zheng Z, Khushalani JS, et al. Annual out-of-pocket expenditures and financial hardship among cancer survivors aged 18-64 years-United States, 20112016. MMWR Morb Mortal Wkly Rep. 2019;68:494-9.

109. Pantziarka P, Bouche G, Meheus L, Sukhatme V, Sukhatme VP, Vikas P. The repurposing drugs in oncology (ReDO) project. Ecancermedicalscience. 2014;8:442.

110. Sasaki J, Ramesh R, Chada S, Gomyo Y, Roth JA, Mukhopadhyay T. The anthelmintic drug mebendazole induces mitotic arrest and apoptosis by depolymerizing tubulin in non-small cell lung cancer cells. Mol Cancer Ther. 2002;1:1201-9.

111. Mukhopadhyay T, Sasaki J, Ramesh R, Roth JA. Mebendazole elicits a potent antitumor effect on human cancer cell lines both in vitro and in vivo. Clin Cancer Res. 2002;8:2963-9.

112. Martarelli D, Pompei P, Baldi C, Mazzoni G. Mebendazole inhibits growth of human adrenocortical carcinoma cell lines implanted in nude mice. Cancer Chemother Pharmacol. 2008;61:809-17.

113. Doudican N, Rodriguez A, Osman I, Orlow SJ. Mebendazole induces apoptosis via Bcl-2 inactivation in chemoresistant melanoma cells. Mol Cancer Res. 2008;6:1308-15.

114. Bai RY, Staedtke V, Aprhys CM, Gallia GL, Riggins GJ. Antiparasitic mebendazole shows survival benefit in 2 preclinical models of glioblastoma multiforme. Neuro Oncol. 2011;13:974-82.

115. Coyne CP, Jones T, Bear R. Gemcitabine-(C4-amide)-[anti-HER2/neu] anti-neoplastic cytotoxicity in dual combination with mebendazole against chemotherapeutic-resistant mammary adenocarcinoma. J Clin Exp Oncol. 2013;2:1000109.

116. Chen XH, Xu YJ, Wang XG, Lin P, Cao BY, Zeng YY, et al. Mebendazole elicits potent antimyeloma activity by inhibiting the USP5/c-Maf axis. Acta Pharmacol Sin. 2019;40:1568-77. 
117. Feng R, Li S, Lu C, Andreas C, Stolz DB, Mapara MY, et al. Targeting the microtubular network as a new antimyeloma strategy. Mol Cancer Ther. 2011;10:1886-96.

118. Niesvizky R, Jayabalan DS, Christos PJ, Furst JR, Naib T, Ely S, et al. BiRD (Biaxin [clarithromycin]/ Revlimid [lenalidomide]/dexamethasone) combination therapy results in high complete- and overallresponse rates in treatment-naive symptomatic multiple myeloma. Blood. 2008;111:1101-9.

119. Ghosh N, Tucker N, Zahurak M, Wozney J, Borrello I, Huff CA. Clarithromycin overcomes resistance to lenalidomide and dexamethasone in multiple myeloma. Am J Hematol. 2014;89:E116-20.

120. Mark TM, Forsberg PA, Rossi AC, Pearse RN, Pekle KA, Perry A, et al. Phase 2 study of clarithromycin, pomalidomide, and dexamethasone in relapsed or refractory multiple myeloma. Blood Adv. 2019; 3:603-11.

121. Demartini G, Esposti D, Marthyn P, Lapidari A, Fraschini F, Scaglione F. Effect of multiple doses of clarithromycin and amoxicillin on IL-6, IFNgamma and IL-10 plasma levels in patients with community acquired pneumonia. J Chemother. 2004;16:82-5.

122. Ding FM, Zhu SL, Shen C, Jiang YQ. Low-dose clarithromycin therapy modulates CD4+ T-cell responses in a mouse model of chronic Pseudomonas aeruginosa lung infection. Respirology. 2012;17:727-34.

123. Nakamura M, Kikukawa Y, Takeya M, Mitsuya H, Hata H. Clarithromycin attenuates autophagy in myeloma cells. Int J Oncol. 2010;37:815-20.

124. Dalva-Aydemir S, Bajpai R, Martinez M, Adekola KU, Kandela I, Wei C, et al. Targeting the metabolic plasticity of multiple myeloma with FDA-approved ritonavir and metformin. Clin Cancer Res. 2015;21:1161-71.

125. Shibata R, Bassermann F, Chandra P, Resemann H, Mazumder A, Pagano M, et al. Ritonavir sensitizes multiple myeloma cells to bortezomib-induced apoptosis. Blood. 2008;112:5159.

126. Driessen C, Müller R, Novak U, Cantoni N, Betticher D, Mach N, et al. Promising activity of nelfinavirbortezomib-dexamethasone in proteasome inhibitor-refractory multiple myeloma. Blood. 2018;132:2097-100.

127. Mendez-Lopez M, Sutter T, Driessen C, Besse L. HIV protease inhibitors for the treatment of multiple myeloma. Clin Adv Hematol Oncol. 2019;17:615-23.

128. Kumar S, Kaufman JL, Gasparetto C, Mikhael J, Vij R, Pegourie B, et al. Efficacy of venetoclax as targeted therapy for relapsed/refractory $t(11 ; 14)$ multiple myeloma. Blood. 2017;130:2401-9.

129. Kaufman JL, Gasparetto C, Schjesvold FH, Moreau P, Touzeau C, Facon T, et al. Targeting BCL-2 with venetoclax and dexamethasone in patients with relapsed/refractory $t(11 ; 14)$ multiple myeloma. Am J Hematol. 2021;96:418-27.

130. Premkumar VJ, Lentzsch S, Pan S, Bhutani D, Richter J, Jagannath S, et al. Venetoclax induces deep hematologic remissions in t $11 ; 14)$ relapsed/refractory AL amyloidosis. Blood Cancer J. 2021;11:10.

131. Kumar SK, Harrison SJ, Cavo M, de la Rubia J, Popat R, Gasparetto C, et al. Venetoclax or placebo in combination with bortezomib and dexamethasone in patients with relapsed or refractory multiple myeloma (BELLINI): a randomised, double-blind, multicentre, phase 3 trial. Lancet Oncol. 2020;21:1630-42. 